\title{
Emergence of Leadership in Second Language Learners' Group Discussion
}

Baikuntha Bhatta

Dokkyo University

\begin{abstract}
.
In group discussion activities among second language learners, it is the equal right of every participant to take a turn-at-talk and take part in the interaction. As the role of the participant is not predefined, every participant is assumed to contribute equally to lead the activity towards its completion. However, as we start observing the occurrences of group discussion tasks, we find something different - a certain participant takes over the role as leader to move the discussion towards the objective of the task. Once a participant starts leading the discussion, the other participants orient to being led and allow themselves to be guided by the leading participant.

Various studies (e.g., Schegloff, 1989; Jacoby \& Gonzales, 1991; Matoesian, 1999) have suggested that the participants' expertise in the discourse might change frequently where they could be seen as more-knowing, or less-knowing. Jacoby and Gonzales (1991) examined the distribution of expertise in a university physics group and highlighted the strategies that the participants apply to show themselves as a more-knowing or less-knowing member of the group. These studies discussed the participants' expertise in relation to the knowledge about the discourse at hand. However, the overall movement of the group task towards completion has not received adequate attention yet.

Using a conversation analytic approach to the turn by turn unfolding of social interaction (Sacks, 1992), this paper aims at analyzing how a particular participant manages to perform the leading role in a task-oriented group discussion with data coming from video recordings of group discussion in second language learning context of Japanese university English classes. It intends to discuss the strategies the participants make use of in guiding their cohorts towards the completion of the task. Basically, the participant who establishes himself as the leader of the group uses five common strategies: (a) taking the initiative to begin the discussion, (b) summarizing what the other participants say, (c) evaluating a co-participant's utterance, (d) reminding the group about the guidelines of the discussion, and (e) explicitly selecting the next speaker to receive their opinions.
\end{abstract}

Keywords: Leadership, Conversation Analysis, Second Language Interaction 